\title{
GENERALIZED BOUNDED VARIATION AND INSERTING POINT MASSES
}

\author{
MANWAH LILIAN WONG
}

\begin{abstract}
Let $d \mu$ be a probability measure on the unit circle and $d \nu$ be the measure formed by adding a pure point to $d \mu$. We give a formula for the Verblunsky coefficients of $d \nu$ following the method of Simon.

Then we consider $d \mu_{0}$, a probability measure on the unit circle with $\ell^{2}$ Verblunsky coefficients $\left(\alpha_{n}\left(d \mu_{0}\right)\right)_{n=0}^{\infty}$ of bounded variation. We insert $m$ pure points $z_{j}$ to $d \mu_{0}$, rescale, and form the probability measure $d \mu_{m}$. We use the formula above to prove that the Verblunsky coefficients of $d \mu_{m}$ are in the form $\alpha_{n}\left(d \mu_{0}\right)+\sum_{j=1}^{m} \frac{\bar{z}_{j}^{n} c_{j}}{n}+E_{n}$, where the $c_{j}$ 's are constants of norm 1 independent of the weights of the pure points and independent of $n$; the error term $E_{n}$ is in the order of $o(1 / n)$. Furthermore, we prove that $d \mu_{m}$ is of $(m+1)$ generalized bounded variation - a notion that we shall introduce in the paper. Then we use this fact to prove that $\lim _{n \rightarrow \infty} \varphi_{n}^{*}\left(z, d \mu_{m}\right)$ is continuous and is equal to $D\left(z, d \mu_{m}\right)^{-1}$ away from the pure points.
\end{abstract}

\section{INTRODUCTION}

Suppose we have a probability measure $d \mu$ on the unit circle $\partial \mathbb{D}=$ $\{z \in \mathbb{C}:|z|=1\}$. We define an inner product and a norm on

Date: February 10th, 2007.

2000 Mathematics Subject Classification. 42C05, 30E10, 05 E35.

Key words and phrases. point masses, bounded variation, generalized bounded variation, decay of Verblunsky coefficients.

* MC 253-37, California Institute of Technology, Pasadena, CA 91125. E-mail: wongmw@caltech.edu . 
$L^{2}(\partial \mathbb{D}, d \mu)$ respectively by

$$
\begin{aligned}
\langle f, g\rangle & =\int_{\partial \mathbb{D}} \overline{f\left(e^{i \theta}\right) g}\left(e^{i \theta}\right) d \mu(\theta) \\
\|f\|_{d \mu} & =\left(\int_{\partial \mathbb{D}}\left|f\left(e^{i \theta}\right)\right|^{2} d \mu(\theta)\right)^{1 / 2}
\end{aligned}
$$

Then we orthogonalize $1, z, z^{2}, \ldots$ to obtain the family of monic orthogonal polynomials associated with the measure $d \mu$, namely, $\left(\Phi_{n}(z, d \mu)\right)_{n=0}^{\infty}$. We denote the normalized family as $\left(\varphi_{n}(z, d \mu)\right)_{n=0}^{\infty}$.

The family of orthogonal polynomials on the unit circle obey the Szegö recursion relation: let $\Phi_{n}^{*}(z)=z^{n} \overline{\Phi_{n}(1 / \bar{z})}$ and $\varphi_{n}^{*}(z)=\Phi_{n}^{*}(z) /\left\|\Phi_{n}\right\|$ (they are often known as the reversed polynomials). Since $\Phi_{n}(z)$ is the unique $n^{\text {th }}$ degree monic polynomial that is orthogonal to $1, z, \ldots, z^{n-1}$, $\Phi_{n}^{*}(z)$ is the unique polynomial of degree $\leq n$ (up to multiplication by a constant) that is orthogonal to $\left\{z, z^{2} \ldots, z^{n}\right\}$. Then we note that $\Phi_{n+1}(z)-z \Phi_{n}(z)$ is a polynomial of degree at most $n$ which is orthogonal to $z, z^{2}, \ldots, z^{n}$, hence, there exists a constant $\alpha_{n}$ such that the following holds

$$
\Phi_{n+1}(z)=z \Phi_{n}(z)-\overline{\alpha_{n}} \Phi_{n}^{*}(z)
$$

$\alpha_{n}$ is called the $n$-th Verblunsky coefficient. From (1.3), we could deduce the following recurrence relation for $\Phi_{n}^{*}$

$$
\Phi_{n+1}^{*}(z)=\Phi_{n}^{*}(z)-\alpha_{n} z \Phi_{n}(z)
$$

Now we consider the norms of the left hand side and the right hand side of (1.3) respectively. First, observe that $\left\|z \Phi_{n}\right\|$ is just $\left\|\Phi_{n}\right\|$. Then note that $\Phi_{n}^{*}(z)$ is of degree strictly less than $n+1$, so it is orthogonal to $\Phi_{n+1}$. Besides, $\left\|\Phi_{n}\right\|=\left\|\Phi_{n}^{*}\right\|$. As a result, we have

$$
\left\|\Phi_{n+1}\right\|^{2}=\left(1-\left|\alpha_{n}\right|^{2}\right)\left\|\Phi_{n}\right\|^{2}=\prod_{j=0}^{n}\left(1-\left|\alpha_{j}\right|^{2}\right)
$$

This also proves that $\alpha_{n} \in \mathbb{D}=\{z \in \mathbb{C}:|z|<1\}$. From (1.3), (1.4) and (1.5) above, we can deduce the Szegö recursion relations for the normalized families as well

$$
\begin{aligned}
& \varphi_{n+1}(z)=\left(1-\left|\alpha_{n}\right|^{2}\right)^{-1 / 2}\left(z \varphi_{n}(z)-\overline{\alpha_{n}} \varphi_{n}^{*}(z)\right) \\
& \varphi_{n+1}^{*}(z)=\left(1-\left|\alpha_{n}\right|^{2}\right)^{-1 / 2}\left(\varphi_{n}^{*}(z)-\alpha_{n} z \varphi_{n}(z)\right)
\end{aligned}
$$


From the arguments above, we see that each non-trivial probability measure on the unit circle $d \mu$ corresponds to a sequence $\left(\alpha_{n}(d \mu)\right)_{n=0}^{\infty}$ in $\mathbb{D}^{\infty}$ called the Verblunsky coefficients. In fact, the reverse is also true by Verblunsky's theorem, i.e., any sequence of complex numbers $\left(a_{n}\right)_{n=0}^{\infty} \in$ $\mathbb{D}^{\infty}$ is the family of Verblunsky coefficients of a unique probability measure on the unit circle. Hence, there is a bijective correspondence between $\left(\alpha_{n}(d \mu)\right)_{n=0}^{\infty}$ and $d \mu$.

The family of Verblunsky coefficients often gives important information about the measure and the family of orthogonal polynomials, for example, from (1.5) we know that $\sum_{j=0}^{\infty}\left|\alpha_{j}\right|^{2}<\infty$ implies that

$$
\lim _{n \rightarrow \infty}\left\|\Phi_{n}\right\|=\prod_{j=0}^{\infty}\left(1-\left|\alpha_{j}\right|^{2}\right)^{1 / 2}>0
$$

This is a fact that we shall use later in the paper. For a more comprehensive introduction to the theory of orthogonal polynomials on the unit circle, the reader should refer to [17, 18], or the classic references [5, 19].

\section{Results}

In this paper we are going to prove three results, the first one being the following formula

Theorem 2.1. Suppose $d \mu$ is a probability measure on the unit circle and $0<\gamma<1$. Let $d \nu$ be the probability measure formed by adding a point mass $\zeta=e^{i \omega} \in \partial \mathbb{D}$ to $d \mu$ in the following manner

$$
d \nu=(1-\gamma) d \mu+\gamma \delta_{\omega}
$$

Then the Verblunsky coefficients of $d \nu$ are given by

$$
\alpha_{n}(d \nu)=\alpha_{n}+\frac{\left(1-\left|\alpha_{n}\right|^{2}\right)^{1 / 2}}{(1-\gamma) \gamma^{-1}+K_{n}(\zeta)} \overline{\varphi_{n+1}(\zeta)} \varphi_{n}^{*}(\zeta)
$$

where

$$
K_{n}(\zeta)=\sum_{j=0}^{n}\left|\varphi_{j}(\zeta)\right|^{2}
$$

and all objects without the label $(d \nu)$ are associated with the measure $d \mu$. 
Before we state the second result, we need to introduce the notion of $p$-generalized bounded variation, $W_{p}\left(\zeta_{1}, \zeta_{2}, \ldots, \zeta_{p}\right)$, which is the class of sequences defined as follows

Definition We say that a sequence $\left(\alpha_{n}\right)_{n=0}^{\infty}$ is of $p$-generalized bounded variation if each $\alpha_{n}$ can be decomposed into $p$ components

$$
\alpha_{n}=\sum_{k=1}^{p} \beta_{n, k}
$$

with $\beta_{n, k} \in \mathbb{C}$ and there exist $\zeta_{1}, \zeta_{2}, \ldots, \zeta_{p} \in \partial \mathbb{D}$ such that for each $1 \leq k \leq p$

$$
\sum_{n=0}^{\infty}\left|\zeta_{k} \beta_{n+1, k}-\beta_{n, k}\right|<\infty
$$

We denote by $W_{p}\left(\zeta_{1}, \zeta_{2}, \ldots, \zeta_{p}\right)$ the class of sequences $\left(\alpha_{n}\right)_{n=0}^{\infty}$ that satisfy (2.4) and (2.5).

In particular, when $p=1$ and $\zeta_{1}=1$, then it becomes the conventional bounded variation. This is why we gave the name $p$-generalized bounded variation.

For the sake of simplicity, we shall write $d \mu \in W_{p}\left(\zeta_{1}, \zeta_{2}, \ldots, \zeta_{p}\right)$ if the family of Verblunsky coefficients of $d \mu$ is in the class $W_{p}\left(\zeta_{1}, \zeta_{2}, \ldots, \zeta_{p}\right)$.

The Szegő function, which will be involved in Theorem 2.2, is defined as follows

Definition If $d \mu=w(\theta) \frac{d \theta}{2 \pi}+d \mu_{s}$ and $\sum_{j=0}^{\infty}\left|\alpha_{j}\right|^{2}<\infty$, the Szegö function is defined as

$$
D(z)=\exp \left(\frac{1}{4 \pi} \int \frac{e^{i \theta}+z}{e^{i \theta}-z} \log w(\theta) d \theta\right)
$$

The well-known Szegö's Theorem asserts the following equality

$$
\prod_{j=0}^{\infty}\left(1-\left|\alpha_{j}\right|^{2}\right)=\exp \left(\int_{0}^{2 \pi} \log (w(\theta)) \frac{d \theta}{2 \pi}\right)
$$

Hence, if $\left(\alpha_{n}\right)$ is $\ell^{2}, \log w(\theta)$ is integrable and $D(z)$ defines an analytic function on $\mathbb{D}$. For a thorough discussion of the Szegö function, the reader may refer to Chapter 2 of [17].

Now we are ready to state the other two results in this paper: 
Theorem 2.2. Let $\zeta_{j}=e^{i \omega_{j}} \in \partial \mathbb{D}, 1 \leq j \leq p$ be distinct. Suppose we have a measure $d \mu$ with $d \mu \in W_{p}\left(\zeta_{1}, \zeta_{2}, \ldots, \zeta_{p}\right)$ such that for each $j$, $\left(\beta_{n, j}\right)_{n=0}^{\infty} \in \ell^{2}$. The following two results hold

(1) For any compact subset $K$ of $\partial \mathbb{D} \backslash\left\{\zeta_{1}, \zeta_{2}, \ldots, \zeta_{p}\right\}$,

$$
\sup _{n ; z \in K}\left|\Phi_{n}^{*}(z)\right|<\infty
$$

(2) The following limits are continuous at $z \neq \zeta_{1}, \zeta_{2}, \ldots, \zeta_{p}$

$$
\begin{aligned}
& \Phi_{\infty}^{*}(z)=\lim _{n \rightarrow \infty} \Phi_{n}^{*}(z)=D(0) D(z)^{-1} \\
& \varphi_{\infty}^{*}(z)=\lim _{n \rightarrow \infty} \varphi_{n}^{*}(z)=D(z)^{-1}
\end{aligned}
$$

and the convergence is uniform on any compact subset $K \subset \partial \mathbb{D} \backslash\left\{\zeta_{1}, \zeta_{2}, \ldots, \zeta_{p}\right\}$. Moreoever, $d \mu_{s}$ is a pure point measure supported on a subset of $\left\{\zeta_{1}, \zeta_{2}, \ldots, \zeta_{p}\right\}$.

Theorem 2.3. Suppose $d \mu_{0} \in W_{1}(1)$ and $\left(\alpha_{n}\left(d \mu_{0}\right)\right)_{n=0}^{\infty} \in \ell^{2}$. We add $m$ distinct pure points $z_{j}=e^{i \omega_{j}}, \omega_{j} \neq 0$, to $d \mu_{0}$ with weights $\gamma_{j}$ to form the probability measure $d \mu_{m}$ as follows

$$
d \mu_{m}=\left(1-\sum_{j=1}^{m} \gamma_{j}\right) d \mu_{0}+\sum_{j=1}^{m} \gamma_{j} \delta_{\omega_{j}}
$$

under the conditions that $0<\gamma_{j}$ and $\sum_{j=1}^{m} \gamma_{j}<1$. Then $d \mu_{m} \in$ $W_{m+1}\left(1, z_{1}, z_{2}, \ldots, z_{m}\right)$ and

$$
\alpha_{n}\left(d \mu_{m}\right)=\alpha_{n}\left(d \mu_{0}\right)+\sum_{j=1}^{m} \frac{{\overline{z_{j}}}^{n} c_{j}}{n}+E_{n}
$$

where $c_{j}=\overline{z_{j}}\left|D\left(z_{j}, d \mu_{0}\right)\right|^{2} D\left(z_{j}, d \mu_{0}\right)^{-2}$ are constants independent of the weights $\gamma_{1}, \gamma_{2}, \ldots, \gamma_{m}$ and of $n$; and

$$
E_{n}=E_{n}\left(z_{1}, z_{2}, \ldots, z_{m}, \gamma_{1}, \gamma_{2}, \ldots, \gamma_{m}\right)=o\left(\frac{1}{n}\right)
$$

Furthermore, for $z \in \partial \mathbb{D} \backslash\left\{1, z_{1}, z_{2}, \ldots, z_{m}\right\}, \varphi_{\infty}^{*}\left(z, d \mu_{m}\right)$ is continuous and is equal to $\left(1-\sum_{j=1}^{m} \gamma_{j}\right)^{-1 / 2} D\left(z, d \mu_{0}\right)^{-1}$. 
Remark: Note that $d \mu_{m_{a . c .}}$ is just $\left(1-\sum_{j=1}^{m} \gamma_{j}\right) d \mu_{0 \text { a.c. }}$ and that $\int \frac{e^{i \theta}+z}{e^{i \theta}-z} \frac{d \theta}{2 \pi}=1$. Hence, $D\left(z, d \mu_{m}\right)=\left(1-\sum_{j=1}^{m} \gamma_{j}\right)^{1 / 2} D\left(z, d \mu_{0}\right)$.

Theorem 2.2 is a generalization of the following result of Nevai [12] and Nikishin [13] which reads

Theorem 2.4. Suppose $\sum_{j=0}^{\infty}\left|\alpha_{j}\right|^{2}<\infty$ and

$$
\sum_{j=0}^{\infty}\left|\alpha_{j+1}-\alpha_{j}\right|<\infty
$$

Then, for any $\delta>0$,

$$
\sup _{n ; \delta<\arg (z)<2 \pi-\delta}\left|\Phi_{n}^{*}(z)\right|<\infty
$$

and away from $z=1$, we have that $\lim _{n \rightarrow \infty} \Phi_{n}^{*}(z)$ exists, is continuous and equal to $D(0) D(z)^{-1}$. Furthermore, $d \mu_{s}=0$ or else a pure point at $z=1$.

The reader may refer to Theorem 10.12.5 of [18] for the proof.

According to Simon [18, the history of the problem is as follows. The earliest work related to adding point masses was done by Wigner-von Neumann [21], where they constructed a potential with an embedded eigenvalue. Later, Gel'fand-Levitan [4] constructed a potential $V$ so that $-\frac{d^{2}}{d x^{2}}+V$ has a spectral measure with a pure point mass at a positive energy and was otherwise equal to the free measure. A more systematic approach to adding point masses to a potential was then taken by Jost-Kohn [6, 7].

Unaware of the Jost-Kohn work and of each other, formulae for adding point masses for orthogonal polynomials on the real line case were found by Uvarov [20] and Nevai [11]. They found the perturbed polynomials, and Nevai computed the perturbed recursion coefficients.

Jost-Kohn theory for orthogonal polynomials on the unit circle appears previously in Cachafeiro-Marcellán [1, 2, 3], Marcellán-Maroni [10], and Peherstorfer-Steinbauer [14]. In particular, if $d \nu$ and $d \mu$ are as defined in (2.1) above, Peherstorfer-Steinbauer [14] proved that boundedness of the first and second kind orthonormal polynomials of $d \mu$ at 
the pure point $\zeta$ implies that $\lim _{n \rightarrow \infty} \alpha_{n}(d \nu)-\alpha_{n}(d \mu)=0$, but they did not establish any rate of convergence.

When I proved (2.2), I was unaware of the following formula found by Geronimus [5]

$$
\Phi_{n}(z, d \nu)=\Phi_{n}(z)-\frac{\Phi_{n}(\zeta) K_{n-1}(z, \zeta)}{(1-\gamma) \gamma^{-1}+K_{n-1}(\zeta, \zeta)}
$$

Years after Geronimus proved (2.16), a similar formula for the real case was rediscovered by Nevai [11], and the same formula for the unit circle case was rediscovered by Cachafeiro-Marcellan [3]. Unaware of Geronimus' result and the fact that Nevai's result also applies to the unit circle, Simon reconsidered this problem and proved formula (3.1) independently using a totally different method (see Theorem 10.13.7 in [18]). However, a more useful form of his result (see formula (3.1) in Section 3) is disguised in his proof and it lays the foundation to Theorem 2.1.

In addition to Nevai, Uvarov and Simon's result mentioned above, we use Prüfer variables as the main tool to prove that $\lim _{n \rightarrow \infty} \Phi_{n}^{*}(z)$ exists. Prüfer variables are named after Prüfer [15]. Their initial introduction in the spectral theory of orthogonal polynomials on the unit circle was made by Nikishin [13] with a significant follow up by Nevai [12]. Both [12] and [13] had results related to Theorem 2.4 and they arrived at the result by essentially the same proof. Later, Prüfer variables were used as a serious tool in spectral theory by Kiselev-Last-Simon [8] and Last-Simon [9].

Most recently, in [17] (Example 1.6.3, p. 72) Simon considered the measure $d \nu$ with one pure point

$$
d \nu=(1-\gamma) \frac{d \theta}{2 \pi}+\gamma \delta_{0}
$$

He proved that the $n$-th degree orthogonal polynomial of $d \nu$ is as follows

$$
\Phi_{n}(z)=z^{n}-\frac{\gamma}{1+(n-1) \gamma}\left(z^{n-1}+z^{n-2}+\cdots+1\right)
$$

and since $\alpha_{n}=-\overline{\Phi_{n+1}(0)}$,

$$
\alpha_{n}(d \nu)=\frac{\gamma}{1+\gamma n} \approx \frac{1}{n}+\frac{1}{\gamma n^{2}}+O\left(\frac{1}{n^{3}}\right)
$$


Here is a sketch of Simon's proof: he considered $L_{n}$, the $(n+1) \times(n+1)$ matrix defined as $\left(L_{n}\right)_{j k}=c_{j-k}$, where $c_{j}=\int e^{-i j \theta} d \mu(\theta)$ is the $j$-th moment of the measure. It is well-known that if $\Phi_{n}(z)=a_{n} z^{n}+$ $a_{n-1} z^{n-1}+\cdots+a_{0}, \delta_{n}=(0,0, \ldots, 0,1)$ and $\langle$,$\rangle being the Euclidean$ norm,

$$
\left(a_{0}, a_{1}, \ldots, a_{n}\right)=\left\langle\delta_{n}, L_{n}^{-1} \delta_{n}\right\rangle^{-1} L_{n}^{-1} \delta_{n}
$$

Therefore, the aim is to compute $L_{n}^{-1}$. By (2.17),$c_{n}=(1-\gamma) \delta_{n 0}+\gamma$. Let $P_{j}$ be the $j \times j$ matrix which is $j^{-1}$ times the matrix of all 1 's, so it is a rank one projection. $L_{n}$ could be decomposed as

$$
L_{n}=(1-\gamma) \mathbf{1}+(n+1) \gamma P_{n+1}
$$

From (2.21), one could deduce that the inverse of $L_{n}$ is

$$
L_{n}^{-1}=(1-\gamma)^{-1}\left(\mathbf{1}-P_{n+1}\right)+(1+n \gamma)^{-1} P_{n+1}
$$

Unfortunately, the method used to prove the result above no longer gives such a nice result when there are two pure points. For instance, we won't have the decomposition as in (2.21), because $L_{n}$ will be a rank $m$ perturbation of $\left(1-\sum_{j=1}^{m} \gamma_{j}\right) \mathbf{1}$ instead, so the computations will be much more complicated. Besides, this method only works for adding one point to $d \theta / 2 \pi$ but fails for more general measures. Therefore, we need another method to attack the problem.

From formula (2.2) we could make a few observations concerning successive Verblunsky coefficients $\alpha_{n+1}(d \nu)$ and $\alpha_{n}(d \nu)$ : first, we use the fact that $\overline{\varphi_{n+1}(\zeta)}=\overline{\zeta^{n+1}} \varphi_{n+1}^{*}(\zeta)$ and rewrite formula (2.2) as

$$
\alpha_{n}(d \nu)=\alpha_{n}+\frac{\left(1-\left|\alpha_{n}\right|^{2}\right)^{1 / 2}}{(1-\gamma) \gamma^{-1}+K_{n}(\zeta)} \overline{\zeta^{n+1}} \varphi_{n+1}^{*}(\zeta) \varphi_{n}^{*}(\zeta)
$$

Let $t_{n}$ be the tail term in the right hand side of (2.23) above. Suppose we can prove that $\varphi_{n}^{*}(\zeta)$ tends to some non-zero limit $L$ as $n$ tends to infinity, then $1 / K_{n}=O(1 / n)$, hence,

$$
\frac{1}{(1-\gamma) \gamma^{-1}+K_{n}(\zeta)}=\frac{1}{K_{n}(\zeta)}+O\left(\frac{1}{n^{2}}\right)
$$

Besides, $\left(\alpha_{n}\right)_{n=0}^{\infty}$ is $\ell^{2}$, therefore $\left(1-\left|\alpha_{n}\right|^{2}\right)^{1 / 2} \rightarrow 1$. As a result,

$$
\alpha_{n}(d \nu)=\alpha_{n}+t_{n} \approx \alpha_{n}+\frac{\overline{\zeta^{n+1}} L^{2}}{n|L|^{2}}+o\left(\frac{1}{n}\right)
$$


Indeed, we shall prove that if $\zeta t_{n+1}-t_{n}$ is summable, by Theorem 2.4, $\lim _{n \rightarrow \infty} \varphi_{n}^{*}\left(z, d \mu_{1}\right)$ exists away from $z=1$. As a result, if we add another a pure point to $d \mu_{1}$, we can use a similar argument to the one above and formula (2.2) to prove that $\alpha_{n}(d \nu)$ is the sum of $\alpha_{n}\left(d \mu_{0}\right)$ plus two tail terms and an error term.

In general, if we have a measure $d \mu_{m}$ as defined in (2.11), then we add one pure point after the other and use formula (2.2) inductively. Therefore, we shall be able to express $\alpha_{n}\left(d \mu_{m}\right)$ as the sum of $\alpha_{n}\left(d \mu_{0}\right)$ plus $m$ tail terms, and an error term

$$
\alpha_{n}\left(d \mu_{m}\right)=\alpha_{n}\left(d \mu_{0}\right)+t_{1, n}+t_{2, n}+\cdots+t_{m, n}+\text { error }
$$

By an argument similar to the one above we observe that $t_{j, n}$ is $O(1 / n)$ and $z_{j} t_{j, n}-t_{j, n-1}$ is small. Of course, the 'smallness' has to be determined by rigorous computations that we shall present in the proof Nonetheless, these observations led us to introduce the notion of generalized bounded variation $W_{m}$, and from that we could deduce that $\lim _{n \rightarrow \infty} \varphi_{n}^{*}\left(z, d \mu_{m}\right)$ exists.

\section{Proof of Theorem 2.1}

In the proof of Theorem 10.13.7 in [18, Simon gave the following formula for the Verblunsky coefficients of $d \nu$

$$
\alpha_{n}(d \nu)=\alpha_{n}-q_{n}^{-1} \bar{\gamma} \overline{\varphi_{n+1}(\zeta)}\left(\sum_{j=0}^{n} \alpha_{j-1} \frac{\left\|\Phi_{n+1}\right\|}{\left\|\Phi_{j}\right\|} \varphi_{j}(\zeta)\right)
$$

where

$$
\begin{aligned}
K_{n}(\zeta) & =\sum_{j=0}^{n}\left|\varphi_{j}(\zeta)\right|^{2} \\
q_{n} & =(1-\gamma)+\gamma K_{n}(\zeta) \\
\alpha_{-1} & =-1
\end{aligned}
$$

and all objects without the label $(d \nu)$ are associated with the measure $d \mu$. 
First, we observe that $\alpha_{j-1}=-\overline{\Phi_{j}(0)}$, therefore, $\alpha_{j-1} /\left\|\Phi_{j}\right\|=-\overline{\varphi_{j}(0)}$. Second, observe that $\left\|\Phi_{n+1}\right\|$ is independent of $j$ so it could be taken out from the summation. As a result, formula (3.1) becomes

$$
\alpha_{n}(d \nu)=\alpha_{n}(d \mu)+q_{n}^{-1} \overline{\gamma \varphi_{n+1}(\zeta)}\left\|\Phi_{n+1}\right\|\left(\sum_{j=0}^{n} \overline{\varphi_{j}(0)} \varphi_{j}(\zeta)\right)
$$

Then we use the Christoffel-Darboux formula, which states that for $x, y \in \mathbb{C}$ with $x \bar{y} \neq 1$,

$$
(1-\bar{x} y)\left(\sum_{j=0}^{n} \overline{\varphi_{j}(x)} \varphi_{j}(y)\right)=\overline{\varphi_{n}^{*}(x)} \varphi_{n}^{*}(y)-\bar{x} \overline{y \varphi_{n}(x)} \varphi_{n}(y)
$$

Besides, note that $q_{n}^{-1} \gamma=\left((1-\gamma) \gamma^{-1}+K_{n}(\zeta)\right)^{-1}$ As a result, (3.5) could be simplified as follows

$$
\alpha_{n}(d \nu)=\alpha_{n}+\frac{\overline{\varphi_{n+1}(\zeta)} \varphi_{n}^{*}(0) \varphi_{n}^{*}(\zeta)}{(1-\gamma) \gamma^{-1}+K_{n}(\zeta)}\left\|\Phi_{n+1}\right\|
$$

Finally, observe that $\varphi_{n}^{*}(0)=\left\|\Phi_{n}\right\|^{-1}$ and that by (1.5) $),\left\|\Phi_{n+1}\right\| /\left\|\Phi_{n}\right\|=$ $\left(1-\left|\alpha_{n}\right|^{2}\right)^{1 / 2}$. This completes the proof.

\section{Proof of Theorem 2.2}

The technique used in this proof is a generalization of the one used in proving Theorem 2.4. It involves Prüfer variables which are defined as follows

Definition Suppose $z_{0}=e^{i \eta} \in \partial \mathbb{D}$ with $\eta \in[0,2 \pi)$. Define the Prüfer variables by

$$
\Phi_{n}\left(z_{0}\right)=R_{n}\left(z_{0}\right) \exp \left(i\left(n \eta+\theta_{n}\left(z_{0}\right)\right)\right)
$$

where $\theta_{n}$ is determined by $\left|\theta_{n+1}-\theta_{n}\right|<\pi$. Here, $R_{n}(z)=\left|\Phi_{n}(z)\right|>0$, $\theta_{n}$ is real. By the fact that $\Phi_{n}^{*}(z)=z^{n} \overline{\Phi_{n}(z)}$ on $\partial \mathbb{D}$, (4.1) is equivalent to

$$
\Phi_{n}^{*}(z)=R_{n}(z) \exp \left(-i \theta_{n}\right)
$$

Under such definition,

$$
\log \left(\frac{\Phi_{n+1}^{*}}{\Phi_{n}^{*}}\right)=\log \left(1-\alpha_{n} \exp \left(i\left[(n+1) \eta+2 \theta_{n}\right]\right)\right)
$$


For simplicity, we let

$$
a_{n}=\alpha_{n} \exp \left(i\left[(n+1) \eta+2 \theta_{n}\right]\right)
$$

Now write $\log \Phi_{n+1}^{*}$ as a telescoping sum

$$
\log \Phi_{n+1}^{*}(z)=\sum_{j=0}^{n}\left(\log \Phi_{j+1}^{*}(z)-\log \Phi_{j}^{*}(z)\right)=\sum_{j=0}^{n} \log \left(\frac{\Phi_{j+1}^{*}(z)}{\Phi_{j}^{*}(z)}\right)
$$

Note that for $|w| \leq Q<1$, there is a constant $K$ such that

$$
|\log (1-w)-w| \leq K|w|^{2}
$$

Together with (4.3), we have

$$
\log \left(\Phi_{n+1}^{*}(z)\right)=-\sum_{j=0}^{n}\left(a_{j}+L\left(a_{j}\right)\right)
$$

where $\left|L\left(a_{j}\right)\right| \leq K\left|a_{j}\right|^{2}$.

Recall that by assumption, $\left(\alpha_{n}\left(d \mu_{0}\right)\right)_{n=0}^{\infty}$ is $\ell^{2}$. Therefore, by (4.4), $\left(a_{n}\right)_{n=0}^{\infty}$ is also $\ell^{2}$, thus $\sum_{j=0}^{\infty} L\left(a_{j}\right)<\infty$. As a result, in order to prove that $\lim _{n \rightarrow \infty} \Phi_{n}^{*}(z)$ exists, it suffices to prove that $\sum_{j=0}^{\infty} a_{j}$ exists.

Let

$$
h_{n}^{(k)}=\sum_{j=0}^{n-1} \bar{\zeta}_{k}^{j} e^{i j \eta}=\frac{{\overline{\zeta_{k}}}^{n} e^{i n \eta}-1}{\overline{\zeta_{k}} e^{i \eta}-1}
$$

Then

$$
\begin{aligned}
h_{n+1}^{(k)}-h_{n}^{(k)} & ={\overline{\zeta_{k}}}^{n} e^{i n \eta} \\
\text { and }\left|h_{n}^{(k)}\right| & \leq 2\left|\overline{\zeta_{k}} e^{i \eta}-1\right|^{-1}
\end{aligned}
$$

Let $g_{j}=\eta+2 \theta_{j}$ and recall that $\alpha_{n}=\sum_{k=1}^{p} \beta_{n, k}$. By rearranging the order of summation, we get

$$
S_{n}=\sum_{j=0}^{n} \alpha_{j} e^{i\left(j \eta+g_{j}\right)}=\sum_{j=0}^{n}\left(\sum_{k=1}^{p} \beta_{j, k}\right) e^{i\left(j \eta+g_{j}\right)}=\sum_{k=1}^{p} B_{n}^{(k)}
$$

where

$$
B_{n}^{(k)}=\sum_{j=0}^{n} \beta_{j, k} e^{i\left(j \eta+g_{j}\right)}
$$


We are going to sum by parts by Abel's formula. Suppose $\left(a_{j}\right)_{j=0}^{\infty}$ is a sequence, we define

$$
\begin{aligned}
& \left(\delta^{+} a\right)_{j}=a_{j+1}-a_{j} \\
& \left(\delta^{-} a\right)_{j}=a_{j}-a_{j-1}
\end{aligned}
$$

Abel's formula states that

$$
\sum_{j=0}^{n}\left(\delta^{+} a\right)_{j} b_{j}=a_{n+1} b_{n}-a_{0} b_{-1}-\sum_{j=0}^{n} a_{j}\left(\delta^{-} b\right)_{j}
$$

Now we apply Abel's formula to $B_{n}^{(k)}$

$$
\begin{aligned}
B_{n}^{(k)} & =\sum_{j=0}^{n}\left(\delta^{+} h^{(k)}\right)_{j}\left(\zeta_{k}^{j} \beta_{j, k} e^{i g_{j}}\right) \\
& =h_{n+1}^{(k)} \zeta_{k}^{n} \beta_{n, k} e^{i g_{n}}-h_{0}^{(k)} \zeta_{k} \beta_{-1, k} e^{i g_{-1}}-\sum_{j=0}^{n} h_{j}^{(k)} \delta^{-}\left(\zeta_{k}^{j} \beta_{j, k} e^{i g_{j}}\right)_{j}
\end{aligned}
$$

Note that the term $h_{0} \zeta_{k}^{-1} \beta_{-1, k} e^{i g_{-1}}$ will be canceled in (4.16), without loss of generality we may assume it to be 0 .

We want to obtain a bound for $B_{n}^{(k)}$. Observe that

$$
\left|\beta_{n, k}\right| \leq \sum_{q=1}^{n}\left|\beta_{q, k}-\overline{\zeta_{k}} \beta_{q-1, k}\right|+\left|\beta_{0}\right| \leq D_{k}
$$

where

$$
D_{k}=\sum_{q=0}^{\infty}\left|\beta_{q, k}-\overline{\zeta_{k}} \beta_{q-1, k}\right|
$$

is finite because $d \mu \in W_{p}\left(\zeta_{1}, \zeta_{2}, \ldots, \zeta_{p}\right)$.

Next, we use the triangle inequality and $\left|e^{i x}-e^{i y}\right| \leq|x-y|$ to obtain

$$
\begin{aligned}
\left|\delta^{-}\left(\zeta_{k}^{j} \beta_{j, k} e^{i g_{j}}\right)_{j}\right| & \leq\left|\beta_{j, k}\left(e^{i g_{j}}-e^{i g_{j-1}}\right)\right|+\left|\zeta_{k} \beta_{j, k}-\beta_{j-1, k}\right| \\
& \leq\left|\beta_{j, k}\left(\theta_{j}-\theta_{j-1}\right)\right|+\left|\zeta_{k} \beta_{j, k}-\beta_{j-1, k}\right|
\end{aligned}
$$

It has been proven for Prüfer variables (see Corollary 10.12.2 of [18]) that

$$
\left|\theta_{n+1}-\theta_{n}\right|<\frac{\pi}{2}\left|\alpha_{n}\right|\left(1-\left|\alpha_{n}\right|\right)^{-1}
$$


Now recall our assumption that for $1 \leq k \leq p,\left(\beta_{n, k}\right)_{n=0}^{\infty}$ is $\ell^{2}$, therefore $\beta_{n, k} \rightarrow 0, \alpha_{n} \rightarrow 0$, which implies $Q=\sup _{n}\left|\alpha_{n}\right|<1$ and $C=\sup _{n}\left(1-\left|\alpha_{n}\right|\right)^{-1}=(1-Q)^{-1}$. For any $n$ we have

$$
\left|B_{n, k}\right| \leq\left|\zeta_{k} e^{i \eta}-1\right|^{-1}\left(2 D_{k}+\frac{\pi}{2} \sum_{j=0}^{\infty}\left|\beta_{j+1, k}\right|\left|\alpha_{j}\right|(1-Q)^{-1}\right)<\infty
$$

It follows that $\sup _{n}\left|S_{n}\right|<\infty$. This proves (2.8).

The computations above also show that the sum in the right hand side of (4.16) is absolutely convergent as $n \rightarrow \infty$ and the convergence is uniform on any compact subset of $\partial \mathbb{D} \backslash\left\{\zeta_{1}, \zeta_{2}, \ldots, \zeta_{p}\right\}$. Therefore, $\lim _{j \rightarrow \infty} \beta_{j, k}=0$ for all $1 \leq k \leq p$ implies that $\lim _{n \rightarrow \infty} B_{n, k}$ exists, thus $\lim _{n \rightarrow \infty} S_{n}$ exists and is finite. This proves (2.10).

Moreover, for each fixed $k,\left(\beta_{n, k}\right)_{n=0}^{\infty}$ is $\ell^{2},\left(\alpha_{n}\right)_{n=0}^{\infty}$ is also $\ell^{2}$, hence the Szegö function $D(z)$ exists and it has boundary values a.e.. Now decompose $d \mu=w(\theta) \frac{d \theta}{2 \pi}+d \mu_{s}$. It is well-known that $\Phi_{n}^{*} \rightarrow D(0) D^{-1}$ in $L^{2}\left(w(\theta) \frac{d \theta}{2 \pi}\right)$. Since $\Phi_{n}^{*} \rightarrow \Phi_{\infty}^{*}$ uniformly on any compact subset of $\partial \mathbb{D} \backslash\left\{\zeta_{1}, \zeta_{2}, \ldots, \zeta_{p}\right\}$, the limit also converges in the $L^{2}$-sense. Besides, it is well known that $D(0)=\lim _{n \rightarrow \infty}\left\|\Phi_{n}\right\|=\prod_{n=0}^{\infty}\left(1-\left|\alpha_{n}\right|^{2}\right)^{1 / 2}$, hence

$$
\begin{aligned}
& \Phi_{\infty}^{*}(z)=D(0) D^{-1}(z) \\
& \varphi_{\infty}^{*}(z)=D^{-1}(z)
\end{aligned}
$$

on $\partial \mathbb{D} \backslash\left\{\zeta_{1}, \zeta_{2}, \ldots, \zeta_{p}\right\}$.

\section{Proof of Theorem 2.3}

We proceed by induction.

5.1. Base Case. Let any object without the label $\left(d \mu_{1}\right)$ be associated with the measure $d \mu_{0}$. First we start by considering adding one pure point $z_{1}=e^{i \omega_{1}} \in \partial \mathbb{D}, \omega_{1} \neq 1$, to $d \mu_{0} \in W_{1}(1)$ which has $\ell^{2}$ Verblunsky coefficients. 
Define $\tilde{\xi}_{n}\left(d \mu_{1}\right)$ as

$$
\tilde{\xi}_{n}\left(d \mu_{1}\right)=\frac{\left(1-\left|\alpha_{n}\right|^{2}\right)^{1 / 2}}{(1-\gamma) \gamma^{-1}+K_{n}\left(z_{1}\right)} \overline{\varphi_{n+1}\left(z_{1}\right)} \varphi_{n}^{*}\left(z_{1}\right)
$$

where $\alpha_{j}=\alpha_{j}\left(d \mu_{0}\right)$ and $\left(\Phi_{n}\right)_{n=0}^{\infty}$ is the family of orthogonal polynomials for $d \mu_{0}$. Because of formula (2.2), we want to simplify $\tilde{\xi}_{n}\left(d \mu_{0}\right)$.

Since $d \mu_{0} \in W_{1}(1)$ and $\sum_{j=0}^{\infty}\left|\alpha_{j}\right|^{2}<\infty$, by Theorem 2.2 $\lim _{n \rightarrow \infty} \varphi_{n}^{*}\left(z_{1}\right)=$ $D\left(z_{1}\right)^{-1}$, which implies $1 / K_{n}\left(z_{1}\right)=O(1 / n)$. Hence,

$$
\tilde{\xi}_{n}\left(d \mu_{1}\right)=\frac{\left(1-\left|\alpha_{n}\right|^{2}\right)^{1 / 2}}{K_{n}\left(z_{1}\right)} \overline{\varphi_{n+1}\left(z_{1}\right)} \varphi_{n}^{*}\left(z_{1}\right)+O\left(\frac{1}{n^{2}}\right)
$$

Moreover, $\overline{\varphi_{n+1}\left(z_{1}\right)}=\overline{z_{1}^{n+1}} \varphi_{n+1}^{*}\left(z_{1}\right)$. We can further simplify and obtain

$$
\alpha_{n}\left(d \mu_{1}\right)=\alpha_{n}+\bar{z}_{1}^{n+1} \frac{D\left(z_{1}\right)^{-2}}{\left|D\left(z_{1}\right)\right|^{-2}} \frac{1}{n}+o\left(\frac{1}{n}\right)
$$

Let $c_{1}=\overline{z_{1}} D\left(z_{1}\right)^{2} /\left|D\left(z_{1}\right)\right|^{2}$. This proves (2.12) for $m=1$.

Remark: Note that the error term in the right hand side of (5.3) is dependent on $\gamma_{1}$. This is because as $\gamma_{0} \rightarrow 0, d \mu_{1} \rightarrow d \mu_{0}$ weakly, which implies that for each $n, \alpha_{n}\left(d \mu_{1}\right) \rightarrow \alpha_{n}\left(d \mu_{0}\right)$. Since the tail term ${\overline{z_{1}}}^{n+1} \frac{D\left(z_{1}\right)^{-2}}{\left.D\left(z_{1}\right)\right|^{-2}} \frac{1}{n}$ in (5.3) is independent of $\gamma_{1}$, if the error term is also independent of $\gamma_{1}, \alpha_{n}\left(d \mu_{1}\right) \not \rightarrow \alpha_{n}\left(d \mu_{0}\right)$.

It remains to show the claimed properties of $\Phi_{n}\left(d \mu_{1}\right)$. To do that, it suffices to show that $\left(\alpha_{n}\left(d \mu_{1}\right)\right)_{n=0}^{\infty}$ is $\ell^{2}$ and it is in the class $W_{2}\left(1, z_{1}\right)$, then we can conclude by Theorem 2.2 .

First of all, it is clear that $\left(\alpha_{n}\left(d \mu_{1}\right)\right)_{n=0}^{\infty}$ is $\ell^{2}$ because $\left(\alpha_{n}\right)_{n=0}^{\infty}$ is $\ell^{2}$ and $\tilde{\xi}_{n}\left(d \mu_{1}\right)$ is $O(1 / n)$.

Next, we want to show that

$$
\sum_{n=0}^{\infty}\left|z_{1} \tilde{\xi}_{n+1}-\tilde{\xi}_{n}\right|<\infty
$$

By (5.2), the error term is in the order of $O\left(1 / n^{2}\right)$, therefore this is the same as showing the following is $\ell^{1}$-summable

$$
\left|\frac{\varphi_{n+2}^{*}\left(z_{1}\right) \varphi_{n+1}^{*}\left(z_{1}\right)\left(1-\left|\alpha_{n+1}\right|^{2}\right)^{1 / 2}}{K_{n+1}}-\frac{\varphi_{n+1}^{*}\left(z_{1}\right) \varphi_{n}^{*}\left(z_{1}\right)\left(1-\left|\alpha_{n}\right|^{2}\right)^{1 / 2}}{K_{n}}\right|
$$

We are going to estimate term by term. 
- Let $\rho_{n}=\left(1-\left|\alpha_{n}\right|^{2}\right)^{1 / 2}$. We estimate the following using the recurrence relation for orthogonal polynomials (1.6)

$$
\begin{aligned}
& \varphi_{n+1}^{*}\left(z_{1}\right)-\varphi_{n}^{*}\left(z_{1}\right) \\
= & \left(\rho_{n} \varphi_{n}^{*}\left(z_{1}\right)-\alpha_{n} \varphi_{n+1}\left(z_{1}\right)\right)-\varphi_{n}^{*}\left(z_{1}\right) \\
= & \left(\rho_{n}-1\right) \varphi_{n}^{*}\left(z_{1}\right)-\alpha_{n} \varphi_{n+1}\left(z_{1}\right)
\end{aligned}
$$

Since $\rho_{n}-1=O\left(\left|\alpha_{n}\right|^{2}\right), \varphi_{n}^{*}\left(z_{1}\right)=D\left(z_{1}\right)^{-1}+o(1)$ and $1 / K_{n}=$ $O(1 / n)$,

$$
\left|\varphi_{n+1}^{*}\left(z_{1}\right)-\varphi_{n}^{*}\left(z_{1}\right)\right|=\left(O\left(\left|\alpha_{n}\right|^{2}\right)+\left|\alpha_{n}\right|\right)\left|D\left(z_{1}\right)^{-1}+o(1)\right|=O\left(\left|\alpha_{n}\right|\right)
$$

Hence,

$$
\left|\frac{\left(\varphi_{n+1}^{*}\left(z_{1}\right)-\varphi_{n}^{*}\left(z_{1}\right)\right) \varphi_{n+1}^{*}\left(z_{1}\right)\left(1-\left|\alpha_{n}\right|^{2}\right)^{1 / 2}}{K_{n}}\right|=O\left(\frac{\left|\alpha_{n}\right|}{n}\right)
$$

- If we change $n$ to $n+1$, the same argument still holds. Therefore,

$$
\left|\frac{\left(\varphi_{n+2}^{*}\left(z_{1}\right)-\varphi_{n+1}^{*}\left(z_{1}\right)\right) \varphi_{n}^{*}\left(z_{1}\right)\left(1-\left|\alpha_{n}\right|^{2}\right)^{1 / 2}}{K_{n}}\right|=O\left(\frac{\left|\alpha_{n+1}\right|}{n}\right)
$$

- Observe that

$$
\left|\left(1-\left|\alpha_{n+1}\right|\right)^{1 / 2}-\left(1-\left|\alpha_{n}\right|\right)^{1 / 2}\right|=O\left(\left|\alpha_{n}\right|+\left|\alpha_{n+1}\right|\right)
$$

Hence,

$$
\left|\frac{\left[\left(1-\left|\alpha_{n+1}\right|\right)^{1 / 2}-\left(1-\left|\alpha_{n}\right|\right)^{1 / 2}\right] \varphi_{n+1}^{*}\left(z_{1}\right) \varphi_{n}^{*}\left(z_{1}\right)}{K_{n}}\right|=O\left(\frac{\left|\alpha_{n+1}\right|+\left|\alpha_{n}\right|}{n}\right)
$$

- Finally, note that

$$
\left(\frac{1}{K_{n+1}}-\frac{1}{K_{n}}\right) \varphi_{n+1}^{*}\left(z_{1}\right) \varphi_{n}^{*}\left(z_{1}\right)\left(1-\left|\alpha_{n}\right|^{2}\right)^{1 / 2}=O\left(\frac{1}{n^{2}}\right)
$$

Combining all the estimates above, we have

$$
\left|z_{1} \tilde{\xi}_{n+1}-\tilde{\xi}_{n}\right|=O\left(\frac{\left|\alpha_{n}\right|+\left|\alpha_{n+1}\right|}{n}\right)+O\left(\frac{1}{n^{2}}\right)
$$

As a result,

$$
\sum_{n=0}^{\infty}\left|z_{1} \tilde{\xi}_{n+1}-\tilde{\xi}_{n}\right|<\infty
$$


and by Theorem 2.2, the proof of the case $m=1$ is complete.

5.2. Induction Step. We consider $d \mu_{m}$ as defined in (2.11) as a measure formed by adding a pure point to $d \mu_{m-1}$ in the following manner

Let

$$
\tilde{\gamma}_{j}=\left(1-\gamma_{m}\right)^{-1} \gamma_{j}
$$

and

$$
d \mu_{m-1}=\left(1-\sum_{l=1}^{m-1} \tilde{\gamma}_{l}\right) d \mu_{0}+\sum_{l=0}^{m-1} \tilde{\gamma}_{l} \delta_{\omega_{l}}
$$

Then we could write

$$
d \mu_{m}=\left(1-\gamma_{m}\right) d \mu_{m-1}+\gamma_{m} \delta_{\omega_{m}}
$$

Recall that $0<\sum_{l=1}^{m} \gamma_{l}<1$, or equivalently, $\sum_{l=1}^{m-1} \gamma_{l}<1-\gamma_{m}$. Hence,

$$
0<\sum_{j=1}^{m-1} \tilde{\gamma}_{j}=\left(1-\gamma_{m}\right)^{-1}\left(\sum_{j=1}^{m-1} \gamma_{j}\right)<1
$$

Therefore, $d \mu_{m-1}$ satisfies the induction hypothesis, so its family of Verblunsky coefficients is $\ell^{2}$ and $d \mu_{m-1} \in W_{m}\left(1, z_{1}, z_{2}, \ldots, z_{m-1}\right)$. Hence, $\lim _{n \rightarrow \infty} \varphi_{n}^{*}\left(z_{m}, d \mu_{m-1}\right)$ exists and is equal to $\left(1-\sum_{j=1}^{m-1} \gamma_{j}\right)^{1 / 2} D\left(z_{m}, d \mu_{0}\right)^{-1}$ (see remark following Theorem 2.3). As a result, we can use a similar argument as in the base case and deduce that

$$
\begin{aligned}
\alpha_{n}\left(d \mu_{m}\right) & =\alpha_{n}\left(d \mu_{m-1}\right)+{\overline{z_{m}}}^{n+1} \frac{\left|D\left(z_{m}, d \mu_{0}\right)\right|^{2}}{D\left(z_{m}, d \mu_{0}\right)^{2}} \frac{1}{n}+E_{n} \\
& =\alpha_{n}\left(d \mu_{0}\right)+\sum_{j=1}^{m} \frac{{\overline{z_{j}}}^{n} c_{j}}{n}+E_{n}
\end{aligned}
$$

where $c_{j}=\overline{z_{j}} D\left(z_{j}, d \mu_{0}\right)^{2} /\left|D\left(z_{j}, d \mu_{0}\right)\right|^{2}, 1 \leq j \leq m$, are constants independent of the weights $\gamma_{1}, \gamma_{2}, \ldots, \gamma_{m}$ and of $n$; and $E_{n}=E_{n}\left(z_{1}, z_{2}, \ldots, z_{m}, \gamma_{1}, \gamma_{2}, \ldots, \gamma_{m}\right)$ is in the order of $o(1 / n)$. This proves (2.12).

By estimating consecutive Verblunsky coefficients in the same way we did in the base case, we prove that $d \mu_{m} \in W_{m+1}\left(1, z_{1}, z_{2}, \ldots, z_{m}\right)$. Thus, we can apply Theorem 2.2 to prove that $\varphi_{n}^{*}\left(z_{m}\right)$ tends to $D\left(z_{m}, d \mu_{m}\right)^{-1}$. This completes the proof of Theorem 2.3 . 
Remark: Note that if $d \mu_{0} \in W_{p}\left(\zeta_{1}, \zeta_{2}, \ldots, \zeta_{p}\right)$ and $z_{j} \neq \zeta_{k}$ for all $j, k$, we can use the same arguments as in the proof of Theorem 2.3 to prove similar results, i.e., $\alpha_{n}\left(d \mu_{m}\right)$ is in the form (2.12), $d \mu_{m}$ is in $W_{m+p}\left(\zeta_{1}, \zeta_{2}, \ldots, \zeta_{p}, z_{1}, z_{2}, \ldots, z_{m}\right)$ and that $\lim _{n \rightarrow \infty} \varphi_{n}\left(z, d \mu_{m}\right)=$ $D\left(z, d \mu_{m}\right)^{-1}$ for $z \neq \zeta_{1}, \zeta_{2}, \ldots, \zeta_{p}, z_{1}, z_{2}, \ldots, z_{m}$.

\section{ACKnowledgements}

I would like to thank Professor Barry Simon for suggesting this problem and proof-reading this paper, as well as his patience and enthusiasm for advising his students; last but not least, for writing the two great reference books [17, 18].

\section{REFERENCES}

[1] A. Cachafeiro and F. Marcellán, Orthogonal polynomials and jump modifications, in "Orthogonal Polynomials and Their Applications", (Segovia, 1986), pp 236-240, Lecture Notes in Math., 1329, Springer, Berlin, 1988

[2] A. Cachafeiro and F. Marcellán, Asymptotics for the ratio of the leading coefficients of orthogonal polynomials associated with a jump modification, in "Approximation and Optimization" (Havana, 1987), pg 111-117, Lecture Notes in Math., 1354, Springer, Berlin, 1988.

[3] A. Cachafeiro and F. Marcellán, Modifications of Toeplitz matrices: jump functions, Rocky Mountain J. Math. 23 (1993), 521-531.

[4] I. M. Gel'fand and B. M. Levitan, On the determination of a differential equation from its spectral function, Amer. Math. Soc. Transl. (2) 1 (1955), 253-304; Russian original in Izvestiya Akad. Nauk SSSR. Ser. Mat. 15 (1951), 309-360.

[5] Ya. L. Geronimus, Polynomials Orthogonal on a Circle and Their Applications, Amer. Math. Soc. Translation 1954 (1954), no. 104, 79pp

[6] R. Jost and W. Kohn, Equivalent potentials, Phys. Rev. 88 (1952), 382-385.

[7] R. Jost and W. Kohn, On the relation between phase shift energy levels and the potential, Danske Vid. Selsk. Mat.-fys/ Medd. 27 (1953), 3-19.

[8] A. Kiselev, Y. Last, and B. Simon, Modified Prüfer and EFGP transforms and the spectral analysis of one-dimensional Schrödinger operators, Comm. Math. Phys. 194 (1998), 1-45.

[9] Y. Last and B. Simon, Eigenfunctions, transfer matrices, and absolutely continuous spectrum of one-dimensional Schödinger operators, Invent. Math. 135 (1999), 329-367. 
[10] F. Marcellán and P. Maroni, Sur l'adjonction d'une masse de Dirac à une forme régulière et semi-classique, Ann. Mat. Pura Appl. (4) 162 (1992), 1-22.

[11] P. Nevai, Orthogonal Polynomials, Mem. Amer. Math. Soc. 18 (1979), no. 213, $185 \mathrm{pp}$.

[12] P. Nevai, Orthogonal polynomials, measures and recurrences on the unit circle, Trans. Amer. Math. Soc. 300 (1987), 175-189.

[13] E.M. Nikishin, An estimate for orthogonal polynomials, Acta Sci. Math. (Szegad) 48 (1985), 395-399. [Russian]

[14] F. Peherstorfer and R. Steinbauer, Mass-points of orthogonality measures on the unit circle, East J. Approx. 5 (1999), 279-308.

[15] H. Prüfer, Neue Herleitung der Sturm-Liouvilleschen Reihenentwicklung stetiger Funktionen, Math. Ann. 95 (1926), 499-518.

[16] B. Simon, OPUC on one foot, Bull. Amer. Math. So. (N.S.) 42 (2005), no. 4, 431-460.

[17] B. Simon, Orthogonal Polynomials on the Unit Circle, Part 1: Classical Theory, AMS Colloquium Series, American Mathematical Society, Providence, RI, 2005.

[18] B. Simon, Orthogonal Polynomials on the Unit Circle, Part 2: Spectral Theory, AMS Colloquium Series, American Mathematical Society, Providence, RI, 2005.

[19] G. Szegő, Orthogonal Polynomials, Amer. Math. Soc. Colloq. Publ., Vol. 23, American Mathematical Society, Providence, R.I., 1939; third edition, 1967.

[20] V.B. Uvarov, The connection between systems of polynomials that are orthogonal with respect to different distribution functions, U.S.S.R. Comput. Math. and Math. Phys. 9 (1969), 1253-1262.

[21] J. von Neumann and E. Wigner, Über merkwürdige diskrete Eigenwerte, Phys. Z. 30 (1929), 465-467.

[22] M.-W. L. Wong, Asymptotics of orthogonal polynomials and point perturbation in a gap, preprint. 